\title{
A NOTE ON STATE SPLITTING
}

\author{
JOEL FRIEDMAN
}

\begin{abstract}
Using state splitting methods, we construct nice matrix representatives for arbitrary subshifts of finite type.
\end{abstract}

In this paper, we will prove the following result.

THEOREM 1. Let $\Sigma_{A}$ be a subshift of finite type (SSFT) with entropy $h\left(\Sigma_{A}\right)$ satisfying

$$
\log n \leq h\left(\Sigma_{A}\right)<\log n+1 .
$$

Then there is an SSFT, $\Sigma_{B}$, topologically conjugate to $\Sigma_{A}$ with

(i) each row sum of $B$ either $n$ or $n+1$,

(ii) the set of column sums of $B$ is the same as that of $A$.

It is known that using the method of symbol splitting (see [ACH and M2]), one can construct SSFT's conjugate to $\Sigma_{A}$ either with all row sums $\geq n$ or with all row sums $\leq n+1$. In our theorem, the inequalities $\geq n$ and $\leq n+1$ are achieved simultaneously. It is known that the above theorem would follow from the lemma below, which was conjectured by Ethan Coven and Brian Marcus at the 1982 AMS Summer Conference in Ergodic Theory (see the methods of $[\mathbf{A C H}$ and $\mathbf{M 1}]$ ).

In addition, given $\Sigma_{A}$ as above, we can first apply the theorem to obtain $\Sigma_{B}$ with row sums $n$ or $n+1$, and then apply the theorem to $\Sigma_{B^{t}}$ to obtain:

THEOREM 2. Let $\Sigma_{A}$ be as in Theorem 1. Then there is an SSFT, conjugate to $\Sigma_{A}$ with each row sum and each column sum either $n$ or $n+1$.

LEMMA. Let $X_{1}, \ldots, X_{k}, n, M$ be positive integers satisfying

$$
\begin{gathered}
\text { each } X_{i} \leq M, \\
\text { some } X_{i}<M, \\
n M \leq \sum_{i=1}^{k} X_{i} \leq(n+1) M .
\end{gathered}
$$

Then there exist positive integers $P_{1}, P_{2}$, and a nontrivial partition of $\{1, \ldots, k\}$ into sets $E_{1}, E_{2}$, such that

$$
M=P_{1}+P_{2}
$$

and

$$
n P_{j} \leq \sum_{i \in E_{j}} X_{i} \leq(n+1) P_{j}, \quad j=1,2 .
$$

Received by the editors October 11, 1983.

1980 Mathematics Subject Classification. Primary 28D20; Secondary 54H20, 58F11. 
Proof. Case 1. $\sum_{i=1}^{k} X_{i}=(n+1) M$. By virtue of (1) and (2), we have $k>n+1$. Consider the sums

$$
X_{1}, X_{1}+X_{2}, \ldots, X_{1}+X_{2}+\cdots+X_{k} .
$$

Since there are $k$ of these sums, two of them must be congruent modulo $n+1$. Their difference is of the form $X_{r}+X_{r+1}+\cdots+X_{s}$, where $1<r \leq s$; this sum is positive, divisible by $n+1$, and does not exceed

$$
\sum_{i=2}^{k} X_{i}=(n+1) M-X_{1}<(n+1) M .
$$

Setting $E_{1}=\{r, r+1, \ldots, s\}, P_{i}=\left(X_{r}+\cdots+X_{s}\right) /(n+1), P_{2}=M-P_{1}$ and $E_{2}=\{1,2, \ldots, k\}-E_{1}$, we have $0<P_{1}<M, P_{1}+P_{2}=M$, and

$$
\sum_{i \in E_{j}} X_{i}=(n+1) P_{j}, \quad j=1,2 .
$$

Hence, this choice of $P_{1}, P_{2}, E_{1}, E_{2}$ satisfies (4) and (5).

Case 2. $\sum_{i=1}^{k} X_{i}<(n+1) M$. Let $\alpha=\left(\sum X_{i}\right) / M$. We have $n \leq \alpha<n+1$. By (1) and (2), we have $k>n$. Consider the sums

$$
X_{1}, X_{1}+X_{2}, \ldots, X_{1}+\cdots+X_{k}
$$

modulo $\alpha$. Since $k \geq n+1>\alpha$, two of these sums must be less than unit distance apart modulo $\alpha$; i.e.,

$$
X_{r}+\cdots+X_{s}=t \alpha+\theta
$$

for integers $r, s, t$ with $1<r \leq s, t<M$, and $\theta$ real with $|\theta|<1$. Let $P_{1}=t$, $E_{1}=\{r, r+1, \ldots, s\}, P_{2}=M-t, E_{2}=\{1, \ldots, k\}-E_{1}$. From (6), it follows that

$$
t \alpha-1<X_{r}+X_{r+1}+\cdots+X_{s}<t \alpha+1,
$$

or

$$
P_{1} \alpha-1<\sum_{i \in E_{1}} X_{i}<P_{1} \alpha+1
$$

and thus

$$
P n-1<\sum_{i \in E_{1}} X<P(n+1)+1 .
$$

Since the expressions in (8) are all integers, it follows that the strict inequalities in (8) imply

$$
P_{1} n \leq \sum_{i \in E_{1}} X_{i} \leq P_{1}(n+1) .
$$

From (7), it follows that $-P_{1} \alpha-1<-\sum_{i \in E_{1}} X_{i}<-P_{1} \alpha+1$, so

$$
-M \alpha+P_{2} \alpha-1<-\sum_{i \in E_{1}} X_{i}<-M \alpha+P_{2} \alpha+1
$$

or $P_{2} \alpha-1<M \alpha-\sum_{i \in E_{1}} X_{i}<P_{2} \alpha+1$ or $P_{2} \alpha-1<\sum_{i \in E_{2}} X_{i}<P_{2} \alpha+1$. Thus, as before,

$$
P_{2} n \leq \sum_{i \in E_{2}} X_{i} \leq P_{2}(n+1) .
$$


By virtue of (9) and (10) we have

$$
\sum_{i \in E_{j}} X_{i} \leq P_{j}(n+1), \quad j=1,2 .
$$

Since $E_{1}$ and $E_{2}$ are nonempty, it follows that $P_{1}$ and $P_{2}$ are positive. Hence, this choice of $P_{1}, P_{2}, E_{1}, E_{2}$ satisfies the desired conditions.

\section{REFERENCES}

[ACH] Roy L. Adler, Don Coopersmith and Martin Hassner, Algorithms for sliding block codes, IEEE Trans. Inform. Theory IT-29 (1983).

[M1] Brian Marcus, Factors and extensions of full shifts, Monatsh. Math. 88 (1979), 239-247.

[M2] _ _ Sofic systems and encoding data (to appear).

IBM RESEARCH LABORATORY, SAN JOSE, CALIFORNIA 95193

Current address: Department of Mathematics, Harvard University, Cambridge, Massachusetts 02138 